\title{
Geometric Representation of the generator of duality in massless and massive $p$-form field theories
}

\author{
Ernesto Contreras ${ }^{1}$, Lorenzo Leal $^{1,2}$ and Yisely Martinez ${ }^{1}$ \\ 1. Grupo de Campos y Partículas, Facultad de Ciencias, Universidad Central de Venezuela, AP 47270, \\ Caracas 1041 - A, Venezuela. \\ 2. Departamento de Física,Universidad Simón Bolivar, AP 89000, \\ Caracas 1080 - A, Venezuela.
}

\begin{abstract}
We study the invariance under duality transformations in massless and massive $p$-form field theories and obtain the Noether generators of the infinitesimal transformations that correspond to this symmetry. These generators are realized in geometrical representations that generalize the Loop Representation of the Maxwell field, allowing for a geometrical interpretation which is studied.
\end{abstract}

\section{INTRODUCTION}

The duality electricity-magnetism that the Maxwell theory exhibits is still a source of inspiration in the search and study of similar transformations in non-Abelian gauge theories, statistical models, string and brane theories and supergravity, among other models. Particular mention deserves the celebrated gauge/string duality [1], that sheds light in the understanding of the non perturbative behavior of gauge theories, which is a mayor challenge of theoretical physics. Apart of its usefulness, duality is a very appealing symmetry: it relates seemingly unrelated models, or switches between different aspects of the same model in a sometimes unexpected fashion.

As it was shown in reference [2, the electric-magnetic duality of the Maxwell theory can be seen as a symmetry of both the equations of motion and the action when the duality transformation is implemented in terms of the Lagrangian variables $A_{\mu}$, in the second order or Lagrangian formulation, or in terms of the variables $A_{\mu}$ and their canonical conjugates in the Hamiltonian or first order formulation. From this point of view, the electricmagnetic duality gives rise to a Noether current, whose associated charge generates the duality rotations of the variables of the theory. More precisely, the $S O(2)$ duality rotations

$$
\left(\begin{array}{c}
E^{\prime i} \\
B^{\prime i}
\end{array}\right)=\left(\begin{array}{cc}
\cos \theta & \operatorname{sen} \theta \\
-\operatorname{sen} \theta & \cos \theta
\end{array}\right)\left(\begin{array}{c}
E^{i} \\
B^{i}
\end{array}\right)
$$

that interchange electric and magnetic fields, or, equivalently, $F^{\mu \nu}$ and its dual $* F^{\mu \nu}$, can be derived from the infinitesimal transformations

$$
\begin{aligned}
& \delta \vec{E}=\beta \nabla \times \vec{A} \\
& \delta \vec{A}=\beta \nabla^{-2} \nabla \times \vec{E}
\end{aligned}
$$

which involve the canonical variables of the theory. It can be seen that the action $S=-\frac{1}{4} \int d^{4} F_{\mu \nu} F^{\mu \nu}$, when written in its first order form, and the equations of motion, are invariant under these infinitesimal transformations (indeed, the action changes by a total time derivative) [2]. The constant of motion obtained from Noether theorem that generates this duality transformation results to be $G=-\frac{1}{2} \int d^{3} x\left(-A^{i} \varepsilon^{i j k} \partial_{j} A^{k}+E^{i} \nabla^{-2} \varepsilon^{i j k} \partial_{j} E^{k}\right)$. 
Models with $p$-form fields, which constitute a generalization of Maxwell theory or Proca theory (depending on wether they are massive or massless), also exhibit a duality symmetry when the dimension of space time and the rank $p$ are appropriately related. These models play an important role in superstring and supergravity theories 3]. Furthermore, $p$-forms couple in a natural way to $(p-1)$-branes, in a straightforward generalization of the coupling of electric or magnetic point charges with the Maxwell field.

In $D$ dimensions, massless theories of ranks $p, q$, with $p+q=D-2$ are mutually dual. For example, in four dimensions, Maxwell theory is self-dual, while the second rank gauge theory is dual to the massles scalar field. Regarding the massive cases, it is found that duality is obtained when the ranks of the forms obey $p+q=D-1$. For instance, the Proca model is self dual in $D=3$.

The realization of electric-magnetic duality as a symmetry of the action, developed in 2] for the Maxwell field, can be also extended to the massless or massive theories of $p$-forms of arbitrary rank mentioned above [4, 5, 6, 7, 8, 9. It is found that the duality group is $Z_{2}$ or $S O(2)$ (in the last case it is then a Noether symmetry) depending on the relationship between the dimension and the rank of the forms involved and on the massive or massless character of the model (see tables (2) and (3) of reference 9]).

In this article, after revisiting the canonical formulation of antisymmetric field theories, we calculate the generator of $S O(2)$ duality transformations for the massless and massive situations, both in the cases of self-duality (i.e., when the theory becomes mapped onto itself under duality rotations) and of duality among two different theories. Then, the theories are quantized in certain geometrical representations that generalize the Loop Representation of the Maxwell field [10, 11, 12, 13] in an appropriate manner. The generators of duality are also realized in these geometrical representation. It is found that the action of the generators onto the wave functionals captures topological invariants of the geometric arguments (the generalized Loops) of these functionals. These invariants are generalized linking numbers and numbers of intersection among $p$-surfaces. This result is a consequence of the fact that the generator of duality is metric-independent, as we shall discuss.

The paper is organized as follows. In sections 2 and 3 we study the canonical quantization and find the generator of duality of massless and massive models of $p$-forms. In section 4 we discuss the path and loop representations [10, 11, 12, 13, present a generalization of this representation to $p$-surfaces [14, 15], and discuss the realization of the generator within this geometrical setting.

\section{MASSLESS MODELS}

\subsection{Self Dual Massless Models}

The action for the theory of a free massless $p$-form field in $D$-dimensional flat space-time can be taken as

$$
S=\frac{(-1)^{p}}{2(p+1) !} \int d^{D} x F_{\mu_{1} \ldots \mu_{p+1}} F^{\mu_{1} \ldots \mu_{p+1}},
$$

where $F_{\mu_{1} \ldots \mu_{p+1}}$ is the field strength defined by

$$
F_{\mu \mu_{1} \ldots \mu_{p}}=\frac{1}{p !} \partial_{[\mu} A_{\left.\mu_{1} \ldots \mu_{p}\right]},
$$

and the $p$-form $A_{\mu_{1} \ldots \mu_{p}}$ is the antisymmetric potential. The equations of motion are given by

$$
\partial_{\mu} F^{\mu \mu_{1} \ldots \mu_{p}}=0 .
$$

When $D-2=2 p$ the theory is self-dual, since equation (2) can be written down as

$$
\partial_{\mu} * F^{\mu \mu_{1} \ldots \mu_{p}}=0,
$$


and the dual field strength $* F^{\mu \mu_{1} \ldots \mu_{p}}=\frac{1}{(p+1) !} \varepsilon^{\mu \mu_{1} \ldots \mu_{p} \nu_{1} \ldots \nu_{p+1}} F_{\nu_{1} \ldots \nu_{p+1}}$ is also a $(p+1)$-form. To display the duality symmetry in terms of the generalized electric and magnetic fields $E^{i_{1} \ldots i_{p}}=F^{i_{1} \ldots i_{p} 0}$ and $B^{i_{1} \ldots i_{p}}=$ $\frac{(-1)^{p}}{(p+1) !} \varepsilon^{i_{1} \ldots i_{p} j_{1} \ldots j_{p+1}} F_{j_{1} \ldots j_{p+1}}$, we expand equations (3) and (4) in the form

$$
\begin{aligned}
\partial_{i} E^{i i_{1} \ldots i_{p-1}} & =0, \\
-\partial_{0} E^{i_{1} \ldots i_{p}}+\frac{1}{p !} \varepsilon^{i_{1} \ldots i_{p} j j_{1} \ldots j_{p}} \partial_{j} B^{j_{1} \ldots j_{p}} & =0, \\
\partial_{j} B^{j j_{1} \ldots j_{p-1}} & =0, \\
(-1)^{p} \partial_{0} B^{i_{1} \ldots i_{p}}-\frac{1}{p !} \varepsilon^{i_{1} \ldots i_{p} j_{1} \ldots j_{p}} \partial_{j} E^{j_{1} \ldots j_{p}} & =0 .
\end{aligned}
$$

As it was studied in references [2, 4, 5, 9, when $p$ is even the equations of motion are invariant under the $Z_{2}$ transformations

$$
\left(\begin{array}{l}
E^{\prime i_{1} \ldots i_{p}} \\
B^{\prime i_{1} \ldots i_{p}}
\end{array}\right)=\left(\begin{array}{ll}
0 & 1 \\
1 & 0
\end{array}\right)\left(\begin{array}{l}
E^{i_{1} \ldots i_{p}} \\
B^{i_{1} \ldots i_{p}}
\end{array}\right)
$$

whereas if $p$ is odd, the equations of motion are left invariant under $S O(2)$ duality rotations

$$
\left(\begin{array}{l}
E^{\prime i_{1} \ldots i_{p}} \\
B^{\prime i_{1} \ldots i_{p}}
\end{array}\right)=\left(\begin{array}{cc}
\cos \theta & \operatorname{sen} \theta \\
-\operatorname{sen} \theta & \cos \theta
\end{array}\right)\left(\begin{array}{c}
E^{i_{1} \ldots i_{p}} \\
B^{i_{1} \ldots i_{p}}
\end{array}\right) \text {. }
$$

In the spirit of reference 2], we are interested in the study of the invariance under duality transformations within the Hamiltonian framework. Hence, we must carry out the canonical analysis of the theory. Following Dirac's procedure for dealing with singular Lagrangeans [16, 17] we obtain the constraints

$$
\begin{aligned}
& \phi_{1}=E^{i_{1} \ldots i_{p-1} 0}=0, \\
& \phi_{2}=\partial_{i_{1}} E^{i_{1} \ldots i_{p}}=0, \\
& \phi_{3}=A_{i_{1} \ldots i_{p-1} 0}=0 \\
& \phi_{4}=\partial_{i_{1}} A_{i_{1} \ldots i_{p}}=0 .
\end{aligned}
$$

The first and second constraints are first class ones, arising from the very definition of the momenta and from the time preservation of the vanishing momenta. The third and fourth are gauge-fixing constraints. Hence, the whole list constitutes a set of second-class constraints.

We are interested in the cases where the duality symmetry is continuous, which correspond to $p$ odd. In this case, the first order action results to be

$$
\begin{aligned}
S & =\int d^{D} x\left(\frac{1}{p !} E^{i_{1} \ldots i_{p}} \dot{A}_{i_{1} \ldots i_{p}}-\frac{1}{2 p !} E^{i_{1} \ldots i_{p}} E^{i_{1} \ldots i_{p}}\right. \\
& \left.-\frac{1}{2(p+1) !} F_{i_{1} \ldots i_{p+1}} F^{i_{1} \ldots i_{p+1}}\right) .
\end{aligned}
$$

It can be verified that varying this action we obtain the canonical equations of motion, in terms of the gauge-fixed canonical variables.

The Dirac brackets among the canonical variables are given by

$$
\begin{aligned}
& \left\{A_{i_{1} \ldots i_{p}}(\vec{x}), E^{j_{1} \ldots j_{p}}(\vec{y})\right\}^{*}=\delta_{i_{1} \ldots i_{p}}^{j_{1} \ldots j_{p}} \delta^{D-1}(\vec{x}-\vec{y})-(p !)^{2} \partial_{i_{1}}^{\vec{x}} \partial_{j_{1}}^{\vec{y}}\left(\delta_{b i_{2} \ldots i_{p}}^{a j_{2} \ldots j_{p}} \partial_{a} \partial_{b}\right)^{-1} \delta^{D-1}(\vec{x}-\vec{y}), \\
& \left\{A_{i_{1} \ldots i_{p}}(\vec{x}), A_{j_{1} \ldots j_{p}}(\vec{y})\right\}^{*}=\left\{E^{i_{1} \ldots j_{p}}(\vec{x}), E^{j_{1} \ldots j_{p}}(\vec{y})\right\}^{*}=0,
\end{aligned}
$$


where $\delta_{i_{1} \ldots i_{p}}^{j_{1} \ldots j_{p}}$ is the antisymmetric Kronecker delta, whose value is +1 if $j_{1} \ldots j_{p}$ is an even permutation of $i_{1} \ldots i_{p}$, -1 if $j_{1} \ldots j_{p}$ is an odd permutation of $i_{1} \ldots i_{p}$ and 0 in the remaining cases. The left hand side of the expression for the bracket among the potential $A$ and its momentum, generalizes the transverse Dirac delta that appears in the canonical formulation of Maxwell theory in the Coulomb gauge, as it should be.

When $p$ is odd, the infinitesimal $S O(2)$ transformations corresponding to equation (7) can be written down in terms of the canonical variables $A_{i_{1} \ldots i_{p}}$ and $E^{i_{1} \ldots i_{p}}$ as

$$
\begin{aligned}
\delta E^{i_{1} \ldots i_{p}} & =-\frac{\theta}{p !} \varepsilon^{i_{1} \ldots i_{p} j j_{1} \ldots j_{p}} \partial_{j} A_{j_{1} \ldots j_{p}} \\
\delta A_{i_{1} \ldots i_{p}} & =-\frac{\theta}{p !} \nabla^{-2} \varepsilon^{i_{1} \ldots i_{p} j j_{1} \ldots j_{p}} \partial_{j} E^{j_{1} \ldots j_{p}}
\end{aligned}
$$

It can be seen that the transformations (11) change the first order action by a total time derivative, just as in the usual Maxwell theory [2]. Then, Noether theorem allows us to obtain the generator of infinitesimal duality-rotations, which results to be

$$
\begin{aligned}
G & =\frac{1}{2 p !^{2}} \int d^{D-1} x\left(A_{i_{1} \ldots i_{p}} \varepsilon^{i_{1} \ldots i_{p} j j_{1} \ldots j_{p}} \partial_{j} A_{j_{1} \ldots j_{p}}\right. \\
& \left.-E^{i_{1} \ldots i_{p}} \nabla^{-2} \varepsilon^{i_{1} \ldots i_{p} j j_{1} \ldots j_{p}} \partial_{j} E^{j_{1} \ldots j_{p}}\right) .
\end{aligned}
$$

This expression for the generator includes the four dimensional Maxwell case, which was studied by Deser and Teitelboim [2]. In that case, $p=1$ and we have

$$
G=\frac{1}{2} \int d^{3} x\left(A^{i} \varepsilon^{i j k} \partial_{j} A^{k}-E^{i} \nabla^{-2} \varepsilon^{i j k} \partial_{j} E^{k}\right) .
$$

We shall come back to the four dimensional Maxwell theory when discussing the realization of the theories in the Loop Representation and its generalizations. The Maxwell case will give us some insight about the geometrical interpretation of the generator that will be useful for the other cases under study .

To quantize the theory we promote the canonical variables to operators obeying equal time canonical commutators

$$
\begin{aligned}
& {\left[\hat{A}_{i_{1} \ldots i_{p}}(\vec{x}), \hat{E}^{j_{1} \ldots j_{p}}(\vec{y})\right]=i \delta_{i_{1} \ldots i_{p}}^{j_{1} \ldots j_{p}} \delta^{D-1}(\vec{x}-\vec{y})-i(p !)^{2} \partial_{i_{1}}^{x} \partial_{j_{1}}^{y}\left(\delta_{b i_{2} \ldots i_{p}}^{a j_{2} \ldots j_{p}} \partial_{a} \partial_{b}\right)^{-1} \delta^{D-1}(\vec{x}-\vec{y}),} \\
& {\left[\hat{A}_{i_{1} \ldots i_{p}}(\vec{x}), \hat{A}_{j_{1} \ldots j_{p}}(\vec{y})\right]=\left[\hat{E}^{i_{1} \ldots i_{p}}(\vec{x}), \hat{E}^{j_{1} \ldots j_{p}}(\vec{y})\right]=0,}
\end{aligned}
$$

and take the evolution of physical states to be given by the Schroedinger equation

$$
i \frac{\partial|\Psi\rangle}{\partial t}=\hat{H}|\Psi\rangle
$$

where $\hat{H}=\frac{1}{2 p !} \int d^{D-1} x\left(\hat{E}^{i_{1} \ldots i_{p}} \hat{E}^{i_{1} \ldots i_{p}}+\hat{B}^{i_{1} \ldots i_{p}} \hat{B}^{i_{1} \ldots i_{p}}\right)$ is the Hamiltonian.

\subsection{Dual Massless Models}

In this section we also consider massless theories, but this time we take the action as

$$
S=\frac{(-1)^{p}}{2(p+1) !} \int d^{D} x F_{\mu_{1} \ldots \mu_{p+1}} F^{\mu_{1} \ldots \mu_{p+1}}+\frac{(-1)^{q}}{2(q+1) !} \int d^{D} x G_{\mu_{1} \ldots \mu_{q+1}} G^{\mu_{1} \ldots \mu_{q+1}},
$$


where

$$
\begin{aligned}
& F_{\mu \mu_{1} \ldots \mu_{p}}=\frac{1}{p !} \partial_{[\mu} A_{\left.\mu_{1} \ldots \mu_{p}\right]}, \\
& G_{\mu \mu_{1} \ldots \mu_{q}}=\frac{1}{q !} \partial_{[\mu} C_{\left.\mu_{1} \ldots \mu_{q}\right]},
\end{aligned}
$$

with $A_{\mu_{1} \ldots \mu_{p}}$ and $C_{\mu_{1} \ldots \mu_{q}}$ antisymmetric potentials. The equations of motion are given by

$$
\begin{aligned}
\partial_{\mu} F^{\mu \mu_{1} \ldots \mu_{p}} & =0, \\
\partial_{\mu} G^{\mu \mu_{1} \ldots \mu_{q}} & =0 .
\end{aligned}
$$

The action (16) corresponds to the theory of two massless and uncoupled antisymmetric gauge potentials (a $p$ and a $q$-form). In references [7, 8, 9, these potentials are seen as the real and imaginary parts of a complex gauge field. The interest in these models rests in the fact that, under certain circumstances, the two intervening theories map into each other when we perform duality transformations. More precisely, for $D-2=p+q$, equations (17) can be written down as

$$
\begin{aligned}
& \partial_{\mu} * F^{\mu \mu_{1} \ldots \mu_{q}}=0, \\
& \partial_{\mu} * G^{\mu \mu_{1} \ldots \mu_{p}}=0,
\end{aligned}
$$

showing, together with equations (18), the duality between the $F$ and $G$ fields.

As before, to study the duality transformations in terms of the generalized electric fields $E^{i_{1} \ldots i_{p}}=F^{i_{1} \ldots i_{p} 0}$ and $D^{i_{1} \ldots i_{q}}=G^{i_{1} \ldots i_{q} 0}$ and generalized magnetic fields $B^{i_{1} \ldots i_{q}}=\frac{(-1)^{p}}{(p+1) !} \varepsilon^{i_{1} \ldots i_{q} j_{1} \ldots j_{p+1}} F_{j_{1} \ldots j_{p+1}}$ and $H^{i_{1} \ldots i_{p}}=$ $\frac{(-1)^{q}}{(q+1) !} \varepsilon^{i_{1} \ldots i_{p} j_{1} \ldots j_{q+1}} G_{j_{1} \ldots j_{q+1}}$, we expand equations (18) and (19) to obtain

$$
\begin{aligned}
\partial_{i} E^{i i_{1} \ldots i_{p-1}} & =0, \\
-\partial_{0} E^{i_{1} \ldots i_{p}}+\frac{(-1)^{(p+1)(q+2)}}{q !} \varepsilon^{i_{1} \ldots i_{p} j j_{1} \ldots j_{q}} \partial_{j} B^{j_{1} \ldots j_{q}} & =0, \\
\partial_{j} B^{j j_{1} \ldots j_{p-1}} & =0, \\
(-1)^{p+1} \partial_{0} B^{i_{1} \ldots i_{q}}+\frac{1}{p !} \varepsilon^{i_{1} \ldots i_{q} j j_{1} \ldots j_{p}} \partial_{j} E^{j_{1} \ldots j_{p}} & =0, \\
\partial_{i} D^{i i_{1} \ldots i_{q-1}} & =0, \\
-\partial_{0} D^{i_{1} \ldots i_{q}}+\frac{(-1)^{(q+1)(p+2)}}{p !} \varepsilon^{i_{1} \ldots i_{q} j j_{1} \ldots j_{p}} \partial_{j} H^{j_{1} \ldots j_{p}} & =0, \\
\partial_{j} H^{j j_{1} \ldots j_{q-1}} & =0, \\
(-1)^{q+1} \partial_{0} H^{i_{1} \ldots i_{p}}+\frac{1}{q !} \varepsilon^{i_{1} \ldots i_{p} j j_{1} \ldots j_{q}} \partial_{j} D^{j_{1} \ldots j_{q}} & =0 .
\end{aligned}
$$

When $p$ and $q$ are even, these equations of motion are invariant under the $Z_{2}$ transformations

$$
\left(\begin{array}{l}
E^{\prime i_{1} \ldots i_{p}} \\
H^{\prime i_{1} \ldots i_{p}}
\end{array}\right)=\left(\begin{array}{ll}
0 & 1 \\
1 & 0
\end{array}\right)\left(\begin{array}{l}
E^{i_{1} \ldots i_{p}} \\
H^{i_{1} \ldots i_{p}}
\end{array}\right)
$$

and

$$
\left(\begin{array}{l}
B^{\prime i_{1} \ldots i_{p}} \\
D^{\prime i_{1} \ldots i_{p}}
\end{array}\right)=\left(\begin{array}{ll}
0 & 1 \\
1 & 0
\end{array}\right)\left(\begin{array}{l}
B^{i_{1} \ldots i_{p}} \\
D^{i_{1} \ldots i_{p}}
\end{array}\right)
$$


On the other hand, if $p$ is odd and $q$ is even or odd the equations of motion remain invariant under $S O(2)$ transformations

$$
\left(\begin{array}{l}
E^{\prime i_{1} \ldots i_{p}} \\
H^{\prime i_{1} \ldots i_{p}}
\end{array}\right)=\left(\begin{array}{cc}
\cos \theta & \operatorname{sen} \theta \\
-\operatorname{sen} \theta & \cos \theta
\end{array}\right)\left(\begin{array}{l}
E^{i_{1} \ldots i_{p}} \\
H^{i_{1} \ldots i_{p}}
\end{array}\right)
$$

and

$$
\left(\begin{array}{c}
B^{\prime i_{1} \ldots i_{q}} \\
D^{\prime i_{1} \ldots i_{q}}
\end{array}\right)=\left(\begin{array}{cc}
\cos \theta & (-1)^{q} \operatorname{sen} \theta \\
(-1)^{q+1} \operatorname{sen} \theta & \cos \theta
\end{array}\right)\left(\begin{array}{c}
B^{i_{1} \ldots i_{q}} \\
D^{i_{1} \ldots i_{q}}
\end{array}\right)
$$

These results agree with those obtained in reference [9].

The canonical analysis leads to the following set of first-class constraints and gauge-fixing constraints

$$
\begin{aligned}
\phi_{1}=E^{i_{1} \ldots i_{p-1} 0}=0 & \phi_{5}=A_{i_{1} \ldots i_{p-1} 0}=0 \\
\phi_{2}=D^{i_{1} \ldots i_{q-1} 0}=0 & \phi_{6}=C_{i_{1} \ldots i_{q-1} 0}=0 \\
\phi_{3}=\partial_{i_{1}} E^{i_{1} \ldots i_{p}}=0 & \phi_{7}=\partial_{i_{1}} A_{i_{1} \ldots i_{p}}=0 \\
\phi_{4}=\partial_{i_{1}} D^{i_{1} \ldots i_{q}}=0 & \phi_{8}=\partial_{i_{1}} C_{i_{1} \ldots i_{q}}=0 .
\end{aligned}
$$

Together, they constitute a set of second-class constraints. When $p$ is odd and $q$ is even or odd the first order action is

$$
\begin{aligned}
S=\int d^{D} x\left(\frac{1}{p !} E^{i_{1} \ldots i_{p}} \dot{A}_{i_{1} \ldots i_{p}}+\frac{1}{q !} D^{i_{1} \ldots i_{q}} \dot{C}_{i_{1} \ldots i_{q}}-\frac{1}{2 p !} E^{i_{1} \ldots i_{p}} E^{i_{1} \ldots i_{p}}-\frac{(-1)^{q+1}}{2 p !} D^{i_{1} \ldots i_{q}} D^{i_{1} \ldots i_{q}}\right. \\
\left.-\frac{1}{2(p+1) !} F_{i_{1} \ldots i_{p+1}} F^{i_{1} \ldots i_{p+1}}+\frac{(-1)^{q}}{2(q+1) !} G_{i_{1} \ldots i_{q+1}} G^{i_{1} \ldots i_{q+1}}\right) .
\end{aligned}
$$

The Dirac brackets of the canonical variables are given by

$$
\begin{aligned}
& \left\{A_{i_{1} \ldots i_{p}}(\vec{x}), E^{j_{1} \ldots j_{p}}(\vec{y})\right\}^{*}=\delta_{i_{1} \ldots i_{p}}^{j_{1} \ldots j_{p}} \delta^{D-1}(\vec{x}-\vec{y})-(p !)^{2} \partial_{i_{1}}^{x} \partial_{j_{1}}^{y}\left(\delta_{b i_{2} \ldots i_{p}}^{a j_{2} \ldots j_{p}} \partial_{a} \partial_{b}\right)^{-1} \delta^{D-1}(\vec{x}-\vec{y}), \\
& \left\{A_{i_{1} \ldots i_{p}}(\vec{x}), A_{j_{1} \ldots j_{p}}(\vec{y})\right\}^{*}=\left\{E^{i_{1} \ldots j_{p}}(\vec{x}), E^{j_{1} \ldots j_{p}}(\vec{y})\right\}^{*}=0, \\
& \left\{C_{i_{1} \ldots i_{q}}(\vec{x}), D^{j_{1} \ldots j_{q}}(\vec{y})\right\}^{*}=\delta_{i_{1} \ldots j_{q}}^{j_{1} \ldots j_{q}} \delta^{D-1}(\vec{x}-\vec{y})-(q !)^{2} \partial_{i_{1}}^{x} \partial_{j_{1}}^{y}\left(\delta_{b i_{2} \ldots i_{q}}^{a j_{2} \ldots j_{q}} \partial_{a} \partial_{b}\right)^{-1} \delta^{D-1}(\vec{x}-\vec{y}), \\
& \left\{C_{i_{1} \ldots i_{q}}(\vec{x}), C_{j_{1} \ldots j_{q}}(\vec{y})\right\}^{*}=\left\{D^{i_{1} \ldots j_{p}}(\vec{x}), D^{j_{1} \ldots j_{p}}(\vec{y})\right\}^{*}=0 .
\end{aligned}
$$

As in the previous case, we seek for the infinitesimal form of the $S O(2)$ duality rotations acting on the canonical variables $A_{\mu_{1} \ldots \mu_{p}}$ and $C_{\mu_{1} \ldots \mu_{p}}$ (rather than on the "magnetic fields") and their conjugate momenta. When $p$ is odd and $q$ is even or odd, the infinitesimal duality rotations are given by

$$
\begin{aligned}
\delta E^{i_{1} \ldots i_{p}} & =\theta \frac{1}{q !} \varepsilon^{i_{1} \ldots i_{p} j j_{1} \ldots j_{q}} \partial_{j} C_{j_{1} \ldots j_{q}} \\
\delta C_{i_{1} \ldots i_{q}} & =\theta \frac{1}{p !} \varepsilon^{i_{1} \ldots i_{q} j j_{1} \ldots j_{p}} \nabla^{-2} \partial_{i} E^{j_{1} \ldots j_{p}}, \\
\delta D^{i_{1} \ldots i_{q}} & =\theta \frac{(-1)^{q+1}}{p !} \varepsilon^{i_{1} \ldots i_{q} j j_{1} \ldots j_{p}} \partial_{j} A_{j_{1} \ldots j_{p}}, \\
\delta A_{i_{1} \ldots i_{p}} & =\theta \frac{(-1)^{q}}{q !} \varepsilon^{i_{1} \ldots i_{p} j j_{1} \ldots j_{q}} \nabla^{-2} \partial_{j} D^{j_{1} \ldots j_{q}} .
\end{aligned}
$$


The generator associated with these transformations is

$$
\begin{array}{r}
G=\frac{1}{p ! q !} \int d^{D-1} x\left((-1)^{q} A_{i_{1} \ldots i_{p}} \varepsilon^{i_{1} \ldots i_{p} j j_{1} \ldots j_{q}} \partial_{j} C_{j_{1} \ldots j_{q}}\right. \\
\left.+(-1)^{q+1} E^{i_{1} \ldots i_{p}} \varepsilon^{i_{1} \ldots i_{p} j j_{1} \ldots j_{q}} \nabla^{-2} \partial_{j} D^{j_{1} \ldots j_{q}}\right)
\end{array}
$$

as can be verified.

As an example, let us consider the generator for the "Maxwell-Klein-Gordon" theory in $2+1$ dimensions, which corresponds to setting $p=1$ and $q=0$ in equation (29). In this case the first order action is

$$
S=-\int d^{3} x\left(E^{i} \dot{A}^{i}-D \dot{C}+\frac{1}{2}\left(E^{i} E^{i}+D^{2}+\varepsilon^{i j k} \partial_{j} A^{k} \varepsilon^{i l m} \partial_{j} A^{m}+\partial_{i} C \partial_{i} C\right)\right)
$$

where $C$ is the scalar field and $D$ its conjugate momenta. The generator is then given by

$$
G=-\int d^{3} x\left(A^{i} \varepsilon^{i j} \partial_{j} C+E^{i} \varepsilon^{i j} \nabla^{-2} \partial_{j} D\right) .
$$

Later, we shall take this simple case as a guide to interpret the geometrical content of the generator in an appropriate generalization of the Loop Representation that will be discussed.

To quantize the theory, we promote the canonical variables to operators obeying equal time canonical commutators obtained from the Dirac brackets, and set up a Schroedinger equation with Hamiltonian given by

$$
\begin{aligned}
\hat{H}=\int d^{D-1}( & \frac{(-1)^{p+1}}{2 p !} \hat{E}^{i_{1} \ldots i_{p}} \hat{E}^{i_{1} \ldots i_{p}}+\frac{(-1)^{q+1}}{2 q !} \hat{D}^{i_{1} \ldots i_{q}} \hat{D}^{i_{1} \ldots i_{q}} \\
& \left.-\frac{(-1)^{p}}{2 q !} \hat{B}^{i_{1} \ldots i_{q}} \hat{B}^{i_{1} \ldots i_{q}}-\frac{(-1)^{q}}{2 p !} \hat{H}^{i_{1} \ldots i_{p}} \hat{H}^{i_{1} \ldots i_{p}}\right) .
\end{aligned}
$$

\section{MASSIVE MODELS}

\subsection{Self Dual Massive Models}

In $D$ dimensions, the theory of a massive $p$-form field is described by the action

$$
S=\frac{(-1)^{p}}{2(p+1) !} \int d^{D} x F_{\mu_{1} \ldots \mu_{p+1}} F^{\mu_{1} \ldots \mu_{p+1}}-(-1)^{p} \frac{m^{2}}{2 p !} \int d^{D} x A_{\mu_{1} \ldots \mu_{p}} A^{\mu_{1} \ldots \mu_{p}},
$$

where $m$ is the mass and

$$
F_{\mu \mu_{1} \ldots \mu_{p}}=\frac{1}{p !} \partial_{[\mu} A_{\left.\mu_{1} \ldots \mu_{p}\right]}
$$

with $A_{\mu_{1} \ldots \mu_{p}}$ being the antisymmetric field.

The equations of motion of this theory are given by

$$
\partial_{\mu} F^{\mu \mu_{1} \ldots \mu_{p}}=-m^{2} A^{\mu_{1} \ldots \mu_{p}} .
$$

When $D-1=2 p$ the theory is self-dual, meaning that equation (34) can be written as

$$
\partial_{\mu} * A^{\mu \mu_{1} \ldots \mu_{p}}=(-1)^{p} * F^{\mu_{1} \ldots \mu_{p}},
$$


which has the same form as equation (35). Therefore, in the massive case, we find a duality symmetry between the potential $A$ and the Hodge dual of the field $F$ [18], unlike the massless case, where duality relates the electric and magnetic parts of the field $F$.

Expanding equations (35) and (36) we obtain

$$
\begin{aligned}
\partial_{i} E^{i i_{1} \ldots i_{p-1}} & =-m^{2} A^{i_{1} \ldots i_{p-1} 0}, \\
(-1)^{p} \partial_{0} E^{i_{1} \ldots i_{p}}+\frac{1}{(p+1) !} \varepsilon^{i_{1} \ldots i_{p} i j_{1} \ldots j_{p-1}} \partial_{i} B^{j_{1} \ldots j_{p-1}} & =-m^{2} A^{i_{1} \ldots i_{p}}, \\
\frac{(-1)^{p+1}}{p !} \partial_{i} \varepsilon^{i i_{1} \ldots i_{p-1} j_{1} \ldots j_{p}} A^{j_{1} \ldots j_{p}} & =B^{i_{1} \ldots i_{p-1}}, \\
\frac{(-1)^{p}}{p !} \varepsilon^{i_{1} \ldots i_{p} j_{1} \ldots j_{p}} \partial_{0} A^{j_{1} \ldots j_{p}}-\frac{1}{(p+1) !} \varepsilon^{i_{1} \ldots i_{p} i j_{1} \ldots j_{p-1}} \partial_{i} A^{j_{1} \ldots j_{p-1} 0} & =\frac{1}{p !} \varepsilon^{i_{1} \ldots i_{p} j_{1} \ldots j_{p}} E^{j_{1} \ldots j_{p}},
\end{aligned}
$$

where $E^{i_{1} \ldots i_{p}}=F^{i_{1} \ldots i_{p} 0}$ and $B^{i_{1} \ldots i_{p-1}}=\frac{(-1)^{p}}{(p+1) !} \varepsilon^{i_{1} \ldots i_{p-1} j j_{1} \ldots j_{p}} F_{j j_{1} \ldots j_{p}}$ are the generalized electric and magnetic fields respectively. As in the massless cases, it is found that the equations of motion are invariant under duality transformations (this time between $F^{\mu_{1} \ldots \mu_{p+1}}$ and $A^{\mu_{1} \ldots \mu_{p}}$, rather than between electric and magnetic fields) belonging to the group $Z_{2}$ or $S O(2)$, depending on the evenness of the integer $p$. This fact can be summarized as follows. Let us introduce the objects

$$
\tilde{F}=\left(\begin{array}{c}
E^{i_{1} \ldots i_{p}} \\
B^{i_{1} \ldots i_{p-1}}
\end{array}\right) \text { and } \tilde{A}=\left(\begin{array}{c}
m \varepsilon^{i_{1} \ldots i_{p} j_{1} \ldots j_{p}} A^{j_{1} \ldots j_{p}} \\
-m A^{i_{1} \ldots i_{p-1} 0}
\end{array}\right)
$$

Then, it can be seen that if $p$ is odd the equations of motion are invariant under $Z_{2}$ transformations given by

$$
\left(\begin{array}{c}
\tilde{F}^{\prime} \\
\tilde{A}^{\prime}
\end{array}\right)=\left(\begin{array}{ll}
0 & 1 \\
1 & 0
\end{array}\right)\left(\begin{array}{l}
F \\
A
\end{array}\right) \text {. }
$$

On the other hand, when $p$ is even, the invariance is under $S O(2)$ rotations

$$
\left(\begin{array}{c}
\tilde{F} \\
\tilde{A}
\end{array}\right)=\left(\begin{array}{cc}
\cos \theta & \operatorname{sen} \theta \\
-\operatorname{sen} \theta & \cos \theta
\end{array}\right)\left(\begin{array}{c}
F \\
A
\end{array}\right) .
$$

As before, these results are in agreement with reference [9]. Carrying out the canonical analysis we obtain the second class constraints

$$
\begin{aligned}
\phi_{1} & =E^{i_{1} \ldots i_{p-1} 0}=0, \\
\phi_{2} & =\partial_{i} E^{i i_{1} \ldots i_{p-1}}+A^{i_{1} \ldots i_{p-1} 0}=0,
\end{aligned}
$$

which should be familiar in the case $p=1$, that corresponds to the Proca field. When $p$ is even, which is the case that exibits duality as a Noether symmetry, the first order action results to be

$$
\begin{array}{r}
S=\int d^{D-1} x\left(\frac{1}{p !} E^{i_{1} \ldots i_{p}} \dot{A}_{i_{1} \ldots i_{p}}-\frac{1}{2 p !} E^{i_{1} \ldots i_{p}} E^{i_{1} \ldots i_{p}}-\frac{1}{2(p-1) !} \partial_{i} E^{i i_{1} \ldots i_{p-1}} \partial_{j} E^{j i_{1} \ldots i_{p-1}}\right. \\
\left.+\frac{1}{2(p+1) !} F_{i_{1} \ldots i_{p+1}} F^{i_{1} \ldots i_{p+1}}-\frac{m^{2}}{2 p !} A_{i_{1} \ldots i_{p}} A^{i_{1} \ldots i_{p}}\right) .
\end{array}
$$

The Dirac brackets between the canonical variables are

$$
\begin{aligned}
& \left\{A_{i_{1} \ldots i_{p}}(\vec{x}), E^{i_{1} \ldots i_{p}}(\vec{y})\right\}^{*}=\delta_{j_{1} \ldots j_{p}}^{i_{1} \ldots i_{p}} \delta^{D-1}(\vec{x}-\vec{y}) \\
& \left\{A_{i_{1} \ldots i_{p}}(\vec{x}), A_{i_{1} \ldots i_{p}}(\vec{y})\right\}^{*}=\left\{E^{i_{1} \ldots i_{p}}(\vec{x}), E^{i_{1} \ldots i_{p}}(\vec{y})\right\}^{*}=0
\end{aligned}
$$


as can be verified.

The infinitesimal $S O(2)$ duality transformations of the canonical variables can be written as

$$
\begin{aligned}
\delta E^{i_{1} \ldots i_{p}} & =\theta \frac{m}{p !} \varepsilon^{i_{1} \ldots i_{p} j_{1} \ldots j_{p}} A^{j_{1} \ldots j_{p}}, \\
\delta A^{i_{1} \ldots i_{p}} & =-\theta \frac{1}{m p !} \varepsilon^{i_{1} \ldots i_{p} j_{1} \ldots j_{p}} E^{i_{1} \ldots i_{p}},
\end{aligned}
$$

and the Noether generator associated with these transformations is

$$
G=\int d^{D-1} x\left(\frac{m}{2(p !)^{2}} \varepsilon^{i_{1} \ldots i_{p} j_{1} \ldots j_{p}} A_{i_{1} \ldots i_{p}} A_{j_{1} \ldots j_{p}}+\frac{1}{2 m(p !)^{2}} \varepsilon^{i_{1} \ldots i_{p} j_{1} \ldots j_{p}} E^{i_{1} \ldots i_{p}} E^{j_{1} \ldots j_{p}}\right) .
$$

Once again, in the quantum theory that corresponds to these models the equal time canonical commutators are

obtained from the Dirac brackets, and the dynamics is governed by the Schroedinger equation $i \frac{\partial|\Psi\rangle}{\partial t}=\hat{H}|\Psi\rangle$, with Hamiltonian

$$
\begin{array}{r}
\hat{H}=\int d^{D-1} x\left(\frac{1}{2 p !} \hat{E}^{i_{1} \ldots i_{p}} \hat{E}^{i_{1} \ldots i_{p}}+\frac{1}{2(p-1) !} \partial_{i} \hat{E}^{i i_{1} \ldots i_{p-1}} \partial_{j} \hat{E}^{j i_{1} \ldots i_{p-1}}\right. \\
\left.+\frac{1}{2(p-1) !} \hat{B}^{i_{1} \ldots i_{p-1}} \hat{B}^{i_{1} \ldots i_{p-1}}+\frac{m^{2}}{2 p !} \hat{A}_{i_{1} \ldots i_{p}} \hat{A}^{i_{1} \ldots i_{p}}\right) .
\end{array}
$$

The lowest dimensionality example of these models corresponds to the simple harmonic oscillator. In that case, $D=1$ (the temporal dimension) and $p=0$. The first order action can be obtained by replacing $E$ by $\frac{p}{\sqrt{m_{o s c}}}$, $A$ by $\sqrt{m_{o s c}} x$ and $m$ by $\sqrt{\frac{k}{m_{o s c}}}$, which yields

$$
S=\int d t\left(p \dot{x}-\frac{p^{2}}{2 m_{o s c}}-\frac{1}{2} k x^{2}\right)
$$

where $p$ and $x$ are the canonical variables, $m_{o s c}$ is the oscillator mass and $k$ is the spring constant. Therefore, the generator of duality is given by

$$
G=\frac{1}{2} \sqrt{k m_{o s c}} x^{2}+\frac{1}{2 \sqrt{k m_{o s c}}} p^{2} .
$$

\subsection{Dual Massive Models}

Finally, we consider theories comprising a pair of uncoupled massive $p$ and $q$-forms, that map one into the other under the action of duality transformations. We take the action as

$$
\begin{aligned}
S & =\frac{(-1)^{p}}{2(p+1) !} \int d^{D} x F_{\mu_{1} \ldots \mu_{p+1}} F^{\mu_{1} \ldots \mu_{p+1}}-(-1)^{p} \frac{m^{2}}{2 p !} \int d^{D} x A_{\mu_{1} \ldots \mu_{p}} A^{\mu_{1} \ldots \mu_{p}} \\
& +\frac{(-1)^{q}}{2(q+1) !} \int d^{D} x G_{\mu_{1} \ldots \mu_{q+1}} G^{\mu_{1} \ldots \mu_{q+1}}-(-1)^{q} \frac{m^{2}}{2 q !} \int d^{D} x C_{\mu_{1} \ldots \mu_{q}} C^{\mu_{1} \ldots \mu_{q}},
\end{aligned}
$$

where $m$ is the mass. The field strengths are

$$
\begin{aligned}
& F_{\mu \mu_{1} \ldots \mu_{p}}=\frac{1}{p !} \partial_{[\mu} A_{\left.\mu_{1} \ldots \mu_{p}\right]}, \\
& G_{\mu \mu_{1} \ldots \mu_{q}}=\frac{1}{q !} \partial_{[\mu} C_{\left.\mu_{1} \ldots \mu_{q}\right]},
\end{aligned}
$$


while $A_{\mu_{1} \ldots \mu_{p}}$ and $C_{\mu_{1} \ldots \mu_{q}}$ correspond to the antisymmetric potentials.

This time the equations of motion are given by

$$
\begin{gathered}
\partial_{\mu} F^{\mu \mu_{1} \ldots \mu_{p}}=-m^{2} A^{\mu_{1} \ldots \mu_{p}} \\
\partial_{\mu} G^{\mu \mu_{1} \ldots \mu_{p}}=-m^{2} C^{\mu_{1} \ldots \mu_{q}} .
\end{gathered}
$$

Taken into account that equations (49) may be written as

$$
\begin{gathered}
\partial_{\mu} * A^{\mu \mu_{1} \ldots \mu_{p}}=(-1)^{p} * F^{\mu_{1} \ldots \mu_{p}} \\
\partial_{\mu} * C^{\mu \mu_{1} \ldots \mu_{q}}=(-1)^{q} * F^{\mu_{1} \ldots \mu_{q}},
\end{gathered}
$$

we see that for $D-1=p+q$ there is a duality symmetry between the $p$ and $q$ fields of the theory. As in the previous cases, this symmetry can be studied at the level of the canonical variables by expanding (50) and (51D) to obtain

$$
\begin{aligned}
\partial_{i} E^{i i_{1} \ldots i_{p-1}} & =-m^{2} A^{i_{1} \ldots i_{p-1} 0}, \\
(-1)^{p} \partial_{0} E^{i_{1} \ldots i_{p}}+\frac{1}{(q-1) !} \varepsilon^{i_{1} \ldots i_{p} i j_{1} \ldots j_{q-1}} \partial_{i} B^{j_{1} \ldots j_{q-1}} & =-m^{2} A^{i_{1} \ldots i_{p}}, \\
\frac{(-1)^{p+1}}{p !} \partial_{i} \varepsilon^{i i_{1} \ldots i_{q-1} j_{1} \ldots j_{p}} A^{j_{1} \ldots j_{p}} & =B^{i_{1} \ldots i_{q-1}}, \\
\frac{(-1)^{p}}{p !} \varepsilon^{i_{1} \ldots i_{q} j_{1} \ldots j_{p}} \partial_{0} A^{j_{1} \ldots j_{p}}-\frac{1}{(p-1) !} \varepsilon^{i_{1} \ldots i_{q} i j_{1} \ldots j_{p-1}} \partial_{i} A^{j_{1} \ldots j_{p-1} 0} & =\frac{1}{p !} \varepsilon^{i_{1} \ldots i_{q} j_{1} \ldots j_{p}} E^{j_{1} \ldots j_{p}}, \\
(-1)^{q} \partial_{0} D^{i_{1} \ldots i_{q} \ldots i_{q-1}}+\frac{1}{(q+1) !} \varepsilon^{i_{1} \ldots i_{q} i j_{1} \ldots j_{q-1}} \partial_{i} H^{j_{1} \ldots j_{q-1}} & =-m^{2} A^{i_{1} \ldots i_{q-1} 0}, \\
\frac{(-1)^{q+1}}{q !} \partial_{i} \varepsilon^{i i_{1} \ldots i_{p-1} j_{1} \ldots j_{q}} C^{j_{1} \ldots j_{q}} & =H^{i_{1} \ldots i_{p}-1}, \\
\frac{(-1)^{q}}{q !} \varepsilon^{i_{1} \ldots i_{p} j_{1} \ldots j_{q}} \partial_{0} C^{j_{1} \ldots j_{q}}-\frac{1}{(q-1) !} \varepsilon^{i_{1} \ldots i_{p} i j_{1} \ldots j_{q-1}} \partial_{i} C^{j_{1} \ldots j_{q-1} 0} & =\frac{1}{q !} \varepsilon^{i_{1} \ldots i_{p} j_{1} \ldots j_{q}} D^{j_{1} \ldots j_{q}} .
\end{aligned}
$$

Here, $E^{i_{1} \ldots i_{p}}=F^{i_{1} \ldots i_{p} 0}$ and $D^{i_{1} \ldots i_{q}}=G^{i_{1} \ldots i_{q} 0}$ are the generalized electric fields, while $B^{i_{1} \ldots i_{q-1}}=\frac{(-1)^{p}}{(p+1) !} \varepsilon^{i_{1} \ldots i_{q-1} j j_{1} \ldots j_{p}} F_{j j_{1} \ldots j_{p}}$ and $H^{i_{1} \ldots i_{p-1}}=\frac{(-1)^{q}}{(q+1) !} \varepsilon^{i_{1} \ldots i_{p-1} j j_{1} \ldots j_{q}} G_{j j_{1} \ldots j_{q}}$ are the magnetic ones. Following already familiar steps, we define the objects

$$
\tilde{F}=\left(\begin{array}{c}
E^{i_{1} \ldots i_{p}} \\
B^{i_{1} \ldots i_{q-1}} \\
D^{i_{1} \ldots i_{q-1}} \\
H^{i_{1} \ldots i_{p}}
\end{array}\right) \text { and } \tilde{A}=\left(\begin{array}{c}
m \varepsilon^{i_{1} \ldots i_{p} j_{1} \ldots j_{q}} C^{j_{1} \ldots j_{q}} \\
-m C^{i_{1} \ldots i_{q-1} 0} \\
m \varepsilon^{i_{1} \ldots i_{q} j_{1} \ldots j_{p}} A^{j_{1} \ldots j_{p}} \\
-m A^{i_{1} \ldots i_{p-1}}
\end{array}\right) \text {. }
$$

Then, for $p$ odd and $q$ even or odd the equations of motion are invariant under $Z_{2}$ transformations

$$
\left(\begin{array}{c}
\tilde{F}^{\prime} \\
\tilde{A}^{\prime}
\end{array}\right)=\left(\begin{array}{ll}
0 & 1 \\
1 & 0
\end{array}\right)\left(\begin{array}{c}
F \\
A
\end{array}\right)
$$

while when $p$ and $q$ are even, the invariance is under $S O(2)$ duality rotations

$$
\left(\begin{array}{c}
\tilde{F} \\
\tilde{A}
\end{array}\right)=\left(\begin{array}{cc}
\cos \theta & \operatorname{sen} \theta \\
-\operatorname{sen} \theta & \cos \theta
\end{array}\right)\left(\begin{array}{c}
F \\
A
\end{array}\right) \text {. }
$$


The canonical formulation of Dirac yields the second class constraints

$$
\begin{aligned}
& \phi_{1}=E^{i_{1} \ldots i_{p-1} 0}=0 \\
& \phi_{2}=D^{i_{1} \ldots i_{q-1} 0}=0 \\
& \phi_{3}=\partial_{i} E^{i i_{1} \ldots i_{p-1}}+A^{i_{1} \ldots i_{p-1} 0}=0 \\
& \phi_{4}=\partial_{i} D^{i i_{1} \ldots i_{q-1}}+C^{i_{1} \ldots i_{q-1} 0}=0
\end{aligned}
$$

When $p$ and $q$ are even, which is the interesting case from the point of view of the Noether theorem, the first order action is given by

$$
\begin{aligned}
S & =\int d^{D-1} x\left(\frac{1}{p !} E^{i_{1} \ldots i_{p}} \dot{A}_{i_{1} \ldots i_{p}}-\frac{1}{2 p !} E^{i_{1} \ldots i_{p}} E^{i_{1} \ldots i_{p}}-\frac{1}{2(p-1) !} \partial_{i} E^{i i_{1} \ldots i_{p-1}} \partial_{j} E^{j i_{1} \ldots i_{p-1}}\right. \\
& +\frac{1}{2(p+1) !} F_{i_{1} \ldots i_{p+1}} F^{i_{1} \ldots i_{p+1}}-\frac{m^{2}}{2 p !} A_{i_{1} \ldots i_{p}} A^{i_{1} \ldots i_{p}}+\frac{1}{q !} D^{i_{1} \ldots i_{q}} \dot{C}_{i_{1} \ldots i_{q}}-\frac{1}{2 q !} D^{i_{1} \ldots i_{q}} D^{i_{1} \ldots i_{q}} \\
& -\frac{1}{2(q-1) !} \partial_{i} D^{i i_{1} \ldots i_{q-1}} \partial_{j} D^{j i_{1} \ldots i_{q-1}}+\frac{1}{2(q+1) !} G_{i_{1} \ldots i_{q+1}} G^{i_{1} \ldots i_{q}+1} \\
& \left.-\frac{m^{2}}{2 q !} C_{i_{1} \ldots i_{q}} C^{i_{1} \ldots i_{q}}\right),
\end{aligned}
$$

and the fundamental Dirac brackets are

$$
\begin{aligned}
& \left\{A_{i_{1} \ldots i_{p}}(\vec{x}), E^{j_{1} \ldots j_{p}}(\vec{y})\right\}^{*}=\delta_{j_{1} \ldots j_{p}}^{i_{1} \ldots i_{p}} \delta^{D-1}(\vec{x}-\vec{y}), \\
& \left\{C_{i_{1} \ldots i_{q}}(\vec{x}), D^{j_{1} \ldots j_{q}}(\vec{y})\right\}^{*}=\delta_{j_{1} \ldots j_{p}}^{i_{1} \ldots j_{p}} \delta^{D-1}(\vec{x}-\vec{y}), \\
& \left\{A_{i_{1} \ldots i_{p}}(\vec{x}), A_{j_{1} \ldots j_{p}}(\vec{y})\right\}^{*}=\left\{E^{i_{1} \ldots i_{p}}(\vec{x}), E^{j_{1} \ldots j_{p}}(\vec{y})\right\}^{*}=0, \\
& \left\{C_{i_{1} \ldots i_{q}}(\vec{x}), C_{j_{1} \ldots j_{q}}(\vec{y})\right\}^{*}=\left\{D^{i_{1} \ldots i_{q}}(\vec{x}), D^{j_{1} \ldots j_{q}}(\vec{y})\right\}^{*}=0 .
\end{aligned}
$$

The behavior of the canonical variables under infinitesimal duality rotations can be written as

$$
\begin{aligned}
\delta E^{i_{1} \ldots i_{p}} & =\theta \frac{m}{q !} \varepsilon^{i_{1} \ldots i_{p} j_{1} \ldots j_{q}} C_{j_{1} \ldots j_{q}}, \\
\delta C^{i_{1} \ldots i_{q}} & =-\theta \frac{1}{m p !} \varepsilon^{i_{1} \ldots i_{q} j_{1} \ldots j_{p}} E^{j_{1} \ldots j_{p}}, \\
\delta D^{i_{1} \ldots i_{q}} & =\theta \frac{m}{p !} \varepsilon^{i_{1} \ldots i_{q} j_{1} \ldots j_{p}} A_{j_{1} \ldots j_{p}}, \\
\delta A^{i_{1} \ldots i_{p}} & =-\theta \frac{1}{m q !} \varepsilon^{i_{1} \ldots i_{p} j_{1} \ldots j_{q}} D_{j_{1} \ldots j_{q}} .
\end{aligned}
$$

The Noether charge that generates these transformations is

$$
G=\int d^{D-1} x\left(\frac{m}{p ! q !} \varepsilon^{i_{1} \ldots i_{p} j_{1} \ldots j_{q}} A_{i_{1} \ldots i_{p}} C_{j_{1} \ldots j_{q}}+\frac{1}{m p ! q !} \varepsilon^{i_{1} \ldots i_{p} j_{1} \ldots j_{q}} E^{i_{1} \ldots i_{p}} D^{j_{1} \ldots j_{q}}\right) .
$$

The quantum theory is obtained as in the previous cases. The equal time canonical commutators are based on the Dirac brackets and the dynamics is given by the Schroedinger equation with Hamiltonian

$$
\begin{aligned}
\hat{H} & =\int d^{D-1} x\left(\frac{1}{2 p !} \hat{E}^{i_{1} \ldots i_{p}} \hat{E}^{i_{1} \ldots i_{p}}+\frac{1}{2(p-1) !} \partial_{i} \hat{E}^{i i_{1} \ldots i_{p-1}} \partial_{j} \hat{E}^{j i_{1} \ldots i_{p-1}}+\frac{1}{2(q-1) !} \hat{B}^{i_{1} \ldots i_{q-1}} \hat{B}^{i_{1} \ldots i_{q-1}}\right. \\
& -\frac{m^{2}}{2 p !} \hat{A}_{i_{1} \ldots i_{p}} \hat{A}^{i_{1} \ldots i_{p}}+\frac{1}{2 q !} \hat{D}^{i_{1} \ldots i_{q}} \hat{D}^{i_{1} \ldots i_{q}}+\frac{1}{2(q-1) !} \partial_{i} \hat{D}^{i i_{1} \ldots i_{q-1}} \partial_{j} \hat{D}^{j i_{1} \ldots i_{q-1}} \\
& \left.+\frac{1}{2(p-1) !} \hat{H}^{i_{1} \ldots i_{p-1}} \hat{H}^{i_{1} \ldots i_{p-1}}-\frac{m^{2}}{2 q !} \hat{C}_{i_{1} \ldots i_{q}} \hat{C}^{i_{1} \ldots i_{q}}\right) .
\end{aligned}
$$


This completes our review of duality transformations in Abelian theories. It should be stressed that all this construction is based on the study of the $D=4$ Maxwell case of reference 2. The different aspects of the duality transformations, depending on whether the theory is massive or not, on the number of different fields appearing, and on the order of the fields and the dimension of the space time, can also be found in previous works [7, 8, 9]. However, as far as we know, the explicit form of the generator $G$ for all these cases, and the detailed canonical analysis of the different models, have not been reported previously.

\section{PATH REPRESENTATION AND GENERALIZATIONS}

In this section we discuss the path-space representation and certain generalizations of it adapted to deal with both the massless and massive models discussed in the previous sections. The purpose of this study is to realize the generators of duality transformations of these models in a geometric representation, in order to get some insight into their geometrical meaning.

We begin by recalling that the Abelian path space can be described as the set of certain equivalence classes of curves $\gamma$ in a manifold, which we take as $R^{n}$ [12, 11, 10, 19. The equivalence relation is given in terms of the so called form factor $T^{i}(\vec{x}, \gamma)$ of the curves

$$
T^{i}(\vec{x}, \gamma)=\int_{\gamma} d y^{i} \delta^{n}(\vec{x}-\vec{y})
$$

as follows: $\gamma$ and $\gamma^{\prime}$ are said to be equivalent (i.e., they represent the same path) if their form factors coincide. Closed curves give raise to a subspace of the path space: the loop space. It can be seen that usual composition of curves translates into a composition of paths that endows path space with an Abelian group structure [11]. The path representation arises when one realizes the canonical field operators onto path-dependent wave functionals $\Psi[\gamma]$. We define the path and loop derivatives $\delta_{i}(\vec{x})$ and $\triangle_{i j}(\vec{x})$ by [1]

$$
\begin{aligned}
u^{i} \delta_{i}(\vec{x}) \Psi[\gamma] & \equiv \Psi\left[\gamma \circ u_{\vec{x}}\right]-\Psi[\gamma], \\
\frac{1}{2} \sigma^{i j}(\vec{x}) \triangle_{i j}(\vec{x}) \Psi[\gamma] & =\Psi[\gamma \circ \delta c]-\Psi[\gamma],
\end{aligned}
$$

where $\circ$ denotes the path space product. The derivative $\delta_{i}(\vec{x})\left(\triangle_{i j}(\vec{x})\right)$ measures the change in the pathdependent wave functional when an infinitesimal path $\delta u$ (infinitesimal loop $\delta c$ ) is attached to its argument $\gamma$ at the point $\vec{x}$. It is understood that these changes are considered up to first order in the infinitesimal vector $\vec{u}$ associated with the small path, or in the surface element $\sigma^{i j}=u^{i} v^{j}-u^{j} v^{i}$ (depending on the case) generated by the infinitesimal vectors $\vec{u}$ and $\vec{v}$ that define the small loop $\delta c$. It can be shown that both derivatives are related by

$$
\partial_{i} \delta_{j}(\vec{x})-\partial_{j} \delta_{i}(\vec{x})=\triangle_{i j}(\vec{x})
$$

As an example of how these operators work, we calculate the path derivative of the form factor. One has

$$
\begin{aligned}
T^{i}\left(\vec{x}, \gamma \circ u_{\vec{y}}\right) & =\int_{\gamma \circ u_{\vec{p}}} d z^{i} \delta^{n}(\vec{x}-\vec{y}) \\
& =T^{i}(\vec{x}, \gamma)+u^{j} \delta_{j}^{i} \delta^{n}(\vec{x}-\vec{y}) .
\end{aligned}
$$

Hence

$$
\delta_{i}(\vec{y}) T^{j}(\vec{x}, \gamma)=\delta_{j}^{i} \delta^{n}(\vec{x}-\vec{y}) .
$$


The Abelian path-space construction may be extended to the case of $p$-surfaces $(p>1)$ (see references [19] for the $p=2$ generalization). In these cases the geometric space can be described as a set of equivalence classes of $p$-surfaces $\Sigma$ labeled by the $p$-surface form-factor, which is defined as

$$
T^{i_{1} \ldots i_{p}}(\vec{x}, \Sigma)=\int_{\Sigma} d \Sigma_{\vec{y}}^{i_{1} \ldots i_{p}} \delta^{D-1}(\vec{x}-\vec{y}) .
$$

Here, $d \Sigma^{i_{1} \ldots i_{p}}$ is the surface element of $\Sigma$. The surfaces $\Sigma$ and $\Sigma^{\prime}$ will be considered equivalent if their form factor are the same. As in the $p=1$ case, closed $p$-surfaces give rise to a subspace of the $p$-surface space: the closed $p$-surface space.

Now we can define the open $p$-surface derivative $\delta_{i_{1} \ldots i_{p}}(\vec{x})$

$$
\sigma^{i_{1} \ldots i_{p}} \delta_{i_{1} \ldots i_{p}}(\vec{x}) \Psi[\Sigma] \equiv \Psi\left[\Sigma \circ \sigma_{\vec{x}}\right]-\Psi[\Sigma],
$$

that measures the change of the path-dependent functional $\Psi[\Sigma]$ when an infinitesimal surface of area $\sigma^{i_{1} \ldots i_{p}}$ is appended to its argument $\Sigma$ at the point $\vec{x}$. As in the case of the path derivative, it is understood that these changes are considered up to first order in $\sigma^{i_{1} \ldots i_{p}}$.

Also, we define the closed $p$-surface derivative, $\triangle_{i_{1} \ldots i_{p+1}}(\vec{x})$, which instead of an open surface attaches an infinitesimal closed one to the argument of the functional. It is given by

$$
\sigma^{i_{1} \ldots i_{p+1}} \triangle_{i_{1} \ldots i_{p+1}}(\vec{x}) \Psi[\Sigma] \equiv \Psi[\Sigma \circ \delta \sigma]-\Psi[\Sigma] .
$$

It can be seen that both derivatives are related by

$$
\triangle_{i i_{1} \ldots i_{p}}(\vec{x})=\frac{1}{p !} \partial_{[i} \delta_{\left.i_{1} \ldots i_{p}\right]}(\vec{x}) .
$$

The open $p$-surface derivative of the generalized form factor is then given by

$$
\delta_{j_{1} \ldots j_{p}}(\vec{y}) T^{i_{1} \ldots i_{p}}(\vec{x}, \Sigma)=\delta_{j_{1} \ldots j_{p}}^{i_{1} \ldots i_{p}} \delta^{D-1}(\vec{x}-\vec{y}) .
$$

It should be clear that this construction is nothing but a generalization of the ideas underlying the path-space formulation to the case of extended objects of higher dimensions. Later, we shall briefly refer to an extension of this framework in the opposite sense: the "signed points" space [20], in which the geometrical objects of interest are collections of points rather than paths or surfaces.

With these tools at hand we are ready to represent the operators acting on $p$-surface dependent wave functionals $\Psi[\Sigma]$. For the self-dual massless models we take

$$
\begin{array}{r}
\hat{E}^{i_{1} \ldots i_{p}}(\vec{x})=T^{i_{1} \ldots i_{p}}(\vec{x}, \Sigma) \\
\hat{B}^{i_{1} \ldots i_{p}}(\vec{x})=-\frac{i}{(p+1) !} \varepsilon^{i_{1} \ldots i_{p} j j_{1} \ldots j_{p}} \triangle_{j j_{1} \ldots j_{p}}(\vec{x}) .
\end{array}
$$

It can be shown that this prescription is a representation of the algebra of the basic observables $\hat{B}^{i_{1} \ldots i_{p}}(\vec{x})$ and $\hat{E}^{j_{1} \ldots j_{p}}(\vec{y})$ that arises from equation (14), namely

$$
\left[\hat{B}^{i_{1} \ldots i_{p}}(\vec{x}), \hat{E}^{j_{1} \ldots j_{p}}(\vec{y})\right]=-i \varepsilon^{i_{1} \ldots i_{p} j j_{1} \ldots j_{p}} \partial_{j}^{x} \delta^{D-1}(\vec{x}-\vec{y}) .
$$

It is well known that in the Maxwell case without sources the Gauss Law constraint $\partial_{i} E^{i}=0$ reduces path-space to the loop-space [12. This is so because in the absence of sources, paths, which are quantum Faradays lines, 
have no charge to emanate from or to arrive, and therefore must be closed. In the generalized case, since $E^{i_{1} \ldots i_{p}}$ is also transverse, the $p$-surface space must be also reduced to the subspace of closed $p$-surfaces. On the other hand, all the observables can be written down in terms of the gauge invariant basic observables, which are the (generalized) electric and magnetic fields. This is so even for the duality generator (which is also gauge invariant), which in the geometric representation of closed $p$-surfaces is given by

$$
\begin{aligned}
\hat{G}=-\frac{1}{2 \Omega_{s} p !(p+1) !} \int d^{D-1} x \int & d^{D-1} y \varepsilon^{i_{1} \ldots i_{p} k_{1} \ldots k_{p+1}} \triangle_{k_{1} \ldots k_{p+1}}(\vec{x}) \triangle_{j_{1} \ldots j_{p} j}(\vec{y}) \frac{(x-y)^{j}}{|\vec{x}-\vec{y}|^{D-1}}+ \\
& +\frac{D-3}{2 \Omega_{s}(p !)^{2}} \oint_{\Sigma} d \Sigma_{\vec{x}}^{i_{1} \ldots i_{p}} \oint_{\Sigma} d \Sigma_{\vec{y}}^{j_{1} \ldots j_{p}} \varepsilon^{i_{1} \ldots i_{p} j_{1} \ldots j_{p} j} \frac{(x-y)^{j}}{|\vec{x}-\vec{y}|^{D-1}} .
\end{aligned}
$$

Here, $\Omega_{s}$ is the volume of the $D$-1-dimensional unitary sphere. The second term is the generalized self-linking number [21] of the closed $p$ surface $\Sigma^{i_{1} \ldots i_{p}}$ (recall that $D=2 p+2$ ). In particular, for $p=1$ and $D=4$ we obtain the realization of the duality generator for Maxwell theory in the loop representation

$$
\begin{array}{r}
\hat{G}=-\frac{1}{16 \pi} \int d^{3} x \int d^{3} y \varepsilon^{i j k} \triangle_{j k}(\vec{x}) \triangle_{i l}(\vec{y}) \frac{(x-y)^{l}}{|\vec{x}-\vec{y}|^{3}} \\
-\frac{1}{8 \pi} \oint_{\gamma} d x^{i} \oint_{\gamma} d y^{j} \varepsilon^{i j k} \frac{(x-y)^{k}}{|\vec{x}-\vec{y}|^{3}} .
\end{array}
$$

The second term, which is the loop self-linking number [21, has an intuitive geometrical interpretation. It measures the oriented number of times that the loop $\gamma_{i}$ intercepts any surface bordered by itself. The interpretation of this invariant in the general case is similar: it counts the oriented number of times that the $p$-surface $\Sigma$ cuts any $p+1$-surface whose boundary is $\Sigma$ itself.

We should recall that the existence of this generator holds for $p$ odd. For $p$ even, there is also a generalized loop representation of the massless self-dual models, however, since duality transformations are discrete in that case, there is no room for an infinitesimal generator.

Now we turn our attention to dual massless models. In these cases, the geometric representation must be built in terms of wave functionals $\Psi\left[\Sigma_{p}, \Sigma_{q}\right]$ that depend on two surfaces $\Sigma_{p}, \Sigma_{q}$ of different dimension. Now we have to kinds of surface operators, each acting on one of the arguments $\left(\Sigma_{p}\right.$ or $\left.\Sigma_{q}\right)$ of the wave functional. Then, following similar steps as before, we prescribe the realization

$$
\begin{array}{r}
\hat{E}^{i_{1} \ldots i_{p}}(\vec{x})=T^{i_{1} \ldots i_{p}}\left(\vec{x}, \Sigma_{p}\right), \\
\hat{B}^{i_{1} \ldots i_{q}}(\vec{x})=i \frac{(-1)^{p}}{(p+1) !} \varepsilon^{i_{1} \ldots i_{q} j j_{1} \ldots j_{p}} \triangle_{j j_{1} \ldots j_{p}}(\vec{x}), \\
\hat{D}^{i_{1} \ldots i_{q}}(\vec{x})=T^{i_{1} \ldots i_{q}}\left(\vec{x}, \Sigma_{q}\right), \\
\hat{H}^{i_{1} \ldots i_{p}}(\vec{x})=i \frac{(-1)^{q}}{(q+1) !} \varepsilon^{i_{1} \ldots i_{p} j j_{1} \ldots j_{q}} \triangle_{j j_{1} \ldots j_{q}}(\vec{x}),
\end{array}
$$

which fulfils the algebra of elementary observables (i.e., generalized electric and magnetic fields) obtained from the canonical algebra

$$
\begin{aligned}
& {\left[\hat{B}^{i_{1} \ldots i_{q}}(\vec{x}), \hat{E}^{j_{1} \ldots j_{p}}(\vec{y})\right]=-i \varepsilon^{i_{1} \ldots i_{q} j j_{1} \ldots j_{p}} \partial_{j}^{x} \delta^{D-1}(\vec{x}-\vec{y}),} \\
& {\left[\hat{H}^{i_{1} \ldots i_{p}}(\vec{x}), \hat{D}^{j_{1} \ldots j_{q}}(\vec{y})\right]=-i \varepsilon^{i_{1} \ldots i_{p} j j_{1} \ldots j_{q}} \partial_{j}^{x} \delta^{D-1}(\vec{x}-\vec{y}) .}
\end{aligned}
$$

As before, the $p$-surfaces and $q$-surfaces spaces result to be reduced to the subspaces of closed surfaces, because both $E^{i_{1} \ldots i_{p}}$ and $D^{i_{1} \ldots i_{q}}$ are transverse in virtue of the generalized Gauss laws that they obey $\left(\partial_{i_{1}} E^{i_{1} \ldots i_{p}}=\right.$ 
$\partial_{i_{1}} D^{i_{1} \ldots i_{q}}=0$. On the other hand, the duality generator, in the cases were the duality group is $S O(2)$ (i.e., when $p$ is odd and $q$ is even or odd) is given by

$$
\begin{aligned}
\hat{G}=\frac{(-1)^{q}}{\Omega_{s}(p+1) ! q !} \int d^{D-1} x & \int d^{D-1} y \varepsilon^{j j_{1} \ldots j_{p} k_{1} \ldots k_{q}} \triangle_{j j_{1} \ldots j_{p}}(\vec{y}) \triangle_{k_{1} \ldots k_{q} k}(\vec{x}) \frac{(x-y)^{k}}{|\vec{x}-\vec{y}|^{D-1}} \\
& +\frac{D-3}{\Omega_{s} p ! q !} \oint_{\Sigma} d \Sigma_{\vec{x}}^{i_{1} \ldots i_{p}} \oint_{\Sigma} d \Sigma_{\vec{y}}^{j_{1} \ldots j_{q}} \varepsilon^{i_{1} \ldots i_{p} j_{1} \ldots j_{q} j} \frac{(x-y)^{j}}{|\vec{x}-\vec{y}|^{D-1}} .
\end{aligned}
$$

Again, the second term admits a simple geometrical interpretation. It corresponds to the generalized linking number between the surfaces $\Sigma_{p}$ and $\Sigma_{q}$ [21, that counts the oriented number of times that the $p$-surface and a region enclosed by the $q$-surface intercept in the $p+q$ dimensional space.

Unlike the massless cases, where the basic operators to be realized are the generalized electric and magnetic fields, in the massive ones we have to realize the potentials, together with the electric fields, which are the canonical variables. For the self-dual massive case we set

$$
\begin{gathered}
\hat{E}^{i_{1} \ldots i_{p}}(\vec{x})=T^{i_{1} \ldots i_{p}}(\vec{x}, \Sigma) \\
\hat{A}_{i_{1} \ldots i_{p}}(\vec{x})=i \delta_{i_{1} \ldots i_{p}}(\vec{x}),
\end{gathered}
$$

which satisfy the canonical algebra. In contrast with the massless models, the surface-space involved is not the subspace of closed surfaces, since this time there is no Gauss Law to force the "electric fields" to be transverse. As before, we are specially interested in the generator of duality rotations, which results to be

$$
\begin{aligned}
\hat{G}= & -\frac{m}{2(p !)^{2}} \int d^{D-1} x \varepsilon^{i_{1} \ldots i_{p} j_{1} \ldots j_{p}} \delta(\vec{x})_{i_{1} \ldots i_{p}} \delta(\vec{x})_{j_{1} \ldots j_{p}} \\
& +\frac{\varepsilon^{i_{1} \ldots i_{p} j_{1} \ldots j_{p}}}{2 m(p !)^{2}} \int d \Sigma_{\vec{x}}^{i_{1} \ldots i_{p}} \int d \Sigma_{\vec{y}}^{j_{1} \ldots j_{p}} \delta^{D-1}(x-y) .
\end{aligned}
$$

This time the second term is not a linking number. Instead, it is the self-intersection number of the closed surface $\Sigma_{p}$ [21] which counts the oriented number of times that, in $D-1=2 p$ dimensions, a $p$-surface punctures itself. It should be noticed that for $p$ odd, the "would be duality-generator" vanishes, in agreement with the fact that in that case duality is a discrete symmetry. For instance, for $p=1$, which corresponds to curves, it can be readily seen that this self-intersection number vanishes.

Finally, let us discuss the dual massive cases. Now we set

$$
\begin{aligned}
& \hat{E}^{i_{1} \ldots i_{p}}(\vec{x})=T^{i_{1} \ldots i_{p}}\left(\vec{x}, \Sigma_{p}\right), \\
& \hat{D}^{i_{1} \ldots i_{q}}(\vec{x})=T^{i_{1} \ldots i_{q}}\left(\vec{x}, \Sigma_{q}\right), \\
& \hat{A}_{i_{1} \ldots i_{p}}(\vec{x})=i \delta_{i_{1} \ldots i_{p}}(\vec{x}), \\
& \hat{C}_{i_{1} \ldots i_{q}}(\vec{x})=i \delta_{i_{1} \ldots i_{q}}(\vec{x}) .
\end{aligned}
$$

which realizes the canonical algebra. These operators act onto wave functionals that depend on two different open surfaces $\Sigma_{p}$ and $\Sigma_{q}$ of dimensions $p$ and $q$ respectively. The generator of duality transformations is given by

$$
\begin{aligned}
& \hat{G}=-\frac{m}{p ! q !} \int d^{D-1} x \varepsilon^{i_{1} \ldots i_{p} j_{1} \ldots j_{q}} \delta_{i_{1} \ldots i_{p}}(\vec{x}) \delta_{j_{1} \ldots j_{q}}(\vec{x}) \\
& +\frac{\varepsilon^{i_{1} \ldots i_{p} j_{1} \ldots j_{q}}}{m p ! q !} \int_{\Sigma} d \Sigma_{\vec{x}}^{i_{1} \ldots i_{p}} \int_{\Sigma^{\prime}} d \Sigma_{\vec{x}^{\prime}}^{j_{1} \ldots j_{q}} \delta^{D-1}(\vec{x}-\vec{y}) .
\end{aligned}
$$

This time, the second term measures the number of oriented intersections between the surfaces $\Sigma^{i_{1} \ldots i_{p}}$ and $\Sigma^{j_{1} \ldots j_{q}}$. 
To conclude, let us discuss briefly how to deal with the "loop representation" for 0 -forms. When $p=0$, the canonical operators can be realized in the space of signed points introduced in reference 20] . There, both the Abelian and non-Abelian cases were considered. For our purposes, we shall just take some results that we need to deal with the Abelian situation. We consider the set whose elements are unordered lists $X$ of "signed" points $x^{s}$, where $s= \pm$. A list would be, for instance: $X=x_{1}^{+}, x_{2}^{-}, \ldots, x_{s}^{+}$. The points can be though as boundaries of oriented paths (maybe starting or ending at the spatial infinity), inasmuch as loops can be seen as boundaries of 2- surfaces. The form factor of a list is defined as

$$
T(x, X)=\sum_{a} s_{a} \delta^{n}\left(x-x_{a}\right),
$$

where $s_{a}$ is the sign of the point at position $x_{a}$. Then we declare that two lists of points $X$ and $Y$ are equivalent if

$$
T(x, X)=T(x, Y) .
$$

Now we proceed as before. Considering functionals $\Psi[X]$ that depend on lists, we can define the object $a\left(x^{s}\right)$ which act on $\Psi[X]$ adding a signed point in $X$. In [20] it is shown that we can also define an operator that measures the change of $\Psi[X]$ when an infinitesimal list of points $\delta Y$ is added, by means of

$$
\triangle_{i}(x) \equiv a(x) \frac{\partial}{\partial x^{i}} a(x)^{-1} .
$$

This object, the "dipole derivative", is analogous to the loop derivative in the loop representation.

As an example, we use this representation to realize the duality generator for the "Maxwell-Klein-Gordon" theory in $D=3$, which was discussed at the end of section 2.2. The operators can be realized as

$$
\begin{array}{r}
\hat{E}^{i}(\vec{x})=T^{i}(\vec{x}, \gamma) \\
\hat{B}(\vec{x})=\frac{-i}{2} \varepsilon^{i j} \triangle_{i j}(\vec{x}) \\
\partial_{i} \hat{C}(\vec{x})=i \triangle_{i}(\vec{x}) \\
\hat{D}(\vec{x})=T(\vec{x}, X)
\end{array}
$$

and the generator of duality can be written down as

$$
\hat{G}=\frac{1}{4 \pi} \int d^{2} x \int d^{2} y \varepsilon^{i j} \triangle_{i j}(\vec{y}) \triangle_{k}(\vec{x}) \frac{(\vec{x}-\vec{y})}{|\vec{x}-\vec{y}|}-\frac{1}{2 \pi} \oint_{\gamma} d^{2} x^{j} \sum_{a} \varepsilon^{i j} s_{a} \frac{\left(x_{a}-x\right)^{i}}{\left|\vec{x}_{a}-\vec{x}\right|^{2}}
$$

As in the previous massless cases, the last term measures a "linking number", this time between closed paths and points in a 2-surface. In other words, it detects wether or not the loop encloses a given point, taking into account both the sense (clock our counterclockwise) in which the path surrounds the point, and the "sign" of the point. This term can be rewritten as the intersection number between the loop and open paths emanating from the positive points or ending at the negative ones, in the form

$$
\begin{array}{r}
\hat{G}=\frac{1}{4 \pi} \int d^{2} x \int d^{2} y \varepsilon^{i j} \triangle(\vec{y})_{i j} \triangle(\vec{x})_{k} \frac{(\vec{x}-\vec{y})^{k}}{|\vec{x}-\vec{y}|} \\
-\int_{\gamma} d x^{i} \oint_{c} d y^{j} \varepsilon^{i j} \delta^{2}(\vec{x}-\vec{y}) .
\end{array}
$$




\section{CONCLUDING REMARKS}

It is worth noticing that in all the cases we have studied the generator of duality is a metric independent quantity. In fact, in the massless cases it is given by the sum of two $B F$ terms in the $(D-1)$-dimensional space. In the case of self duality (for instance, in Maxwell theory in $D=4$ ), instead of $B F$ terms we should speak of two Chern-Simons terms: one of them is constructed with the vector potential, the second one with a vector potential for the electric field. In the massive models, the metric independence is also clear: the generator comprises terms which are the integral over the $(D-1)$-dimensional space of $(D-1)$-forms (see equations (44) and (59)). This fact explains why in the geometric representations the generator yields generalized link invariants, as we found.

The topological character of the duality symmetry has also a different (but related) manifestation that we discuss briefly. In reference [22] it was shown that the electric-magnetic duality of Abelian gauge theories induces a description of their physical phase space in terms of a pair of non-local observables that are dual in the Kramers-Wannier sense. The algebra that these observables obey is invariant under spatial diffeomorphisms. For instance, in the case of Maxwell theory in four space time dimensions, the dual operators are the Wilson Loop and the t Hooft disorder operator [5]. In reference [23, this result was also extended to the case of massive theories, and to the case of the Maxwell-Chern-Simons theory and the so called Self-Dual theory, which are dual.

It is interesting to observe that for each model we have studied in this article, there is also a dual geometric representation. For instance, in the Maxwell-Klein-Gordon model in $D=3$, which we realized in the space of electric loops and points associated to the momentum of the scalar field, we could equally well choose to work in a space of magnetic loops and points associated with the scalar field. Then, to interpret the generator of duality we should exchange the roles of the two terms: the one with generalized loops derivatives turns into a term that measures linking numbers, and viceversa. However, we do not have yet a simple interpretation of both terms of the generator in a single geometrical representation. It would also be interesting to study if there is a geometric representation analogous to the ones here considered that allows to realize the generator of duality for linearized gravity, which was study in reference [6] as a generalization of the results of [2. These and other related matters are under study.

\section{References}

[1] J. M. Maldacena, Adv. Theor. Math. Phys. 2, 231 (1998), Int. J. Theor. Phys. 38, 1113 (1999).

[2] S. Deser and C. Teitelboim, Phys.Rev. D13, 1592 (1976).

[3] M. B. Green, J. H. Schwarz and E.Witten, Superstring Theory (Cambridge University Press, Cambridge, 1987).

[4] S. Deser, A. Gomberoff, M. Henneaux, C. Teitelboim, Phys.Lett.B400, 80 (1997), hep-th/9702184.

[5] S. Deser, A. Gomberoff, M. Henneaux, C. Teitelboim, Nucl.Phys.B520:179-204,1998, hep-th/9712189.

[6] M. Henneaux, C. Teitelboim, Phys.Rev.D71, 024018 (2005), gr-qc/0408101.

[7] C. Wotzasek, Nucl.Phys.B(Proc. Suppl.)127:84-91,2004, hep-th/0305127

[8] J.L. Noroha, D. Rocha, M.S. Guimarães and C. Wotzasek,Phys.Rev. B564,163-168,(2003), hep-th/0305102.

[9] R. Menezes and C.Wotzasek,Phys.Lett. B 604 (2004) 242-249, hep-th/0410240

[10] R. Gambini and A. Trias, Phys. Rev. D 27, 2935 (1983) 
[11] R. Gambini and J. Pullin, Loops, Knots, Gauge Theory and Quantum Gravity (Cambridge University Press, 1996)

[12] C. di Bartolo, F. Nori, R. Gambibi and A. Trias, Nuovo Cimento Soc. Ital. Fis. 38,497, 1983.

[13] J. Camacaro, R. Gaitan, L, Leal, Mod.Phys.Lett.A12, 3081 (1997), hep-th/9606121.

[14] P. J. Arias, C. Di Bartolo, X. Fustero, R. Gambini and A. Trias, Int. J. Mod. Phys. A 7, 737 (1992).

[15] P.J. Arias, E. Fuenmayor, L. Leal, Phys.Rev.D69, 125010, (2004), hep-th/0402224.

[16] P. Dirac. Lectures on Quantum Mechanics. Yeshiva University, New York, 1964

[17] M. Henneaux and C. Teitelboim. Quantization of Gauge Systems. Princeton University, New Jersey, 1992.

[18] P.K. Townsend. TH-3067-CERN. April, 1981

[19] L. Leal and A. López, J. Math. Phys, 47(2006) 012305, hep-th/0410258.

[20] L. Leal, Phys. Rev. D 66, 105027 (2002)

[21] D. Rolfsen. Knots and links. Perish,1976.

[22] Lorenzo Leal, Mod.Phys.Lett.A11, 1107 (1996), hep-th/9603006.

[23] Pio J. Arias, Lorenzo Leal, Jean Carlos Perez-Mosquera, Phys.Rev.D67, 025020 (2003), hep-th/0206082. 